\section{Does current thyroid cancer staging accurately reflect the impact of lymph node metastases on survival in younger patients?}

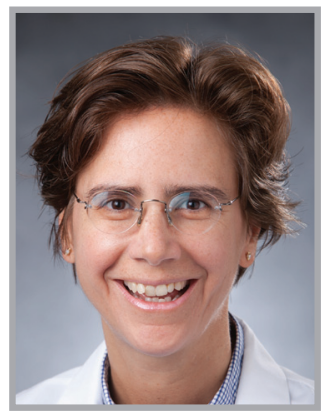

\author{
"Important questions remain: is there an \\ alternative age cut-point to 45 years where \\ prognosis changes? Should we abandon the \\ concept of age dichotomization as a \\ prognostic factor in papillary thyroid \\ cancer altogether? More data are \\ needed in this research space."
}

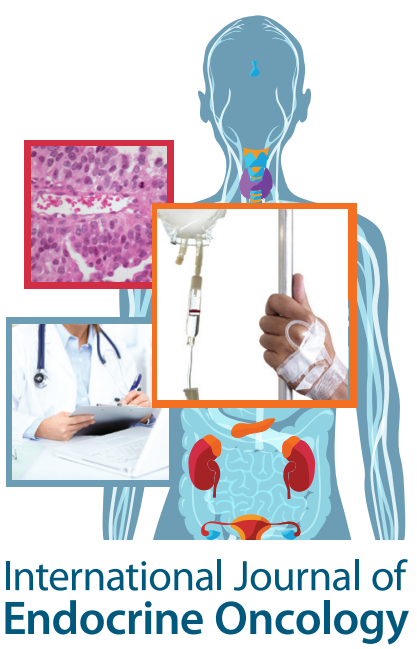

Endocrine Oncology

Mohamed Abdelgadir Adam', Shelby D Reed ${ }^{2}$, Sanziana A Roman' \& Julie A Sosa*1,2

In a recent study published in the Journal of Clinical Oncology (JCO), Adam et al. examined the prognostic role of cervical lymph node metastases among papillary thyroid cancer (PTC) patients who are $<45$ years. They demonstrate that the presence and number of cervical lymph node metastases are associated with compromised survival in patients $<45$ years with Stage I (i.e., without distant metastases) PTC [1]. In a National Cancer Data Base (NCDB) cohort of nearly 50,000 patients $<45$ years at diagnosis, overall survival was compromised for patients with lymph node metastases compared with patients who did not have them. Lymph node metastases were associated with a $32 \%$ increase in risk of death, which is comparable to the reported effect of lymph node metastases on survival among patients $\geq 45$ years. The significant association between lymph node metastases and survival was externally validated in a separate patient cohort from the Surveillance, Epidemiology, and End
Results Database (SEER). An increasing number of metastatic cervical lymph nodes were associated with increasing mortality for up to six metastatic lymph nodes, after which more positive lymph nodes did not appear to confer additional risk of death. This study's findings suggest that rigorous preoperative screening for nodal metastases should be performed for the purposes of informing decisions about surgery and prognosis. These data raise the question of whether the current American Joint Committee on Cancer (AJCC) staging system for PTC may understage young patients harboring lymph node metastases and argue for potential reconsideration of lymph node metastases in a revised AJCC staging system.

Currently, patient age is considered to play a unique prognostic role in the biology and natural history of differentiated thyroid cancer. Indeed, it is the only cancer for which patient age is factored into various staging systems, including the AJCC [2];

\section{KEYWORDS:}

- AJCC • lymph node metastases

- nodal metastases - papillary thyroid cancer • prognosis • survival - thyroid cancer • thyroid cancer staging $\bullet<45$ years $\bullet$ young age

\footnotetext{
"While disease recurrence is an important end point in differentiated thyroid cancer, the observed survival difference in young patients with lymph node metastases is a notable and impactful finding."
} 
the European Organization for Research and Treatment of Cancer (EORTC) [3]; the National Thyroid Cancer Treatment Cooperative Study (NTCTCS) [4]; AGES (age, grade, extent and size) [5]; AMES (age, metastases, extent and size of tumor) [6] and MACIS (metastases, age, completeness of resection, invasion and size) $[1,7,8]$. In the current AJCC staging schema, patients $<45$ years are considered to have a distinctly better prognosis than those $\geq 45$ years. As such, only two stages of disease are available for patients $<45$ years: Stage I disease, defined by the absence of distant, or extracervical, metastases; or Stage II disease, defined by the presence of distant metastases. In contrast, patients $\geq 45$ years can have Stages I-IV disease based on a number of different features, including tumor size, extent of tumor involvement, and the status of lymph node and distant metastases [9]. This distinct age dichotomization at 45 years has been propagated for years based on data from the early- to mid1900 s, which demonstrated a sharp rise in mortality from differentiated thyroid cancer starting around the ages of 40-50 years [10-13]. However, it would seem that these data and their interpretation should be revisited, given that they are old, based on small sample sizes (fewer than 700 patients), and lacked adjustment for important potential confounders.

An age cut-point of 45 years was added to the AJCC staging system (second edition) in 1983 [14]. The prognostic impact of metastatic lymph nodes among patients $\geq 45$ years is well established and has been demonstrated in several studies [15-17]. Among younger patients, the impact of nodal metastases on survival has been more controversial. In a single-institution study of 200 patients with differentiated thyroid cancer, Hughes et al. reported that 20-year survival was compromised for patients $\geq 45$ years with lymph node metastases but not for younger patients [15]. Zaydfudim et al. analyzed data for 15,497 patients with PTC from the SEER database and demonstrated that lymph node metastases were associated with compromised survival among patients $\geq 45$ years; however, the presence of lymph node metastases had no bearing on survival for patients $<45$ years [16]. In a recent analysis of 49,240 patients of all ages with differentiated thyroid cancer, Tran Cao et al. showed that lymph node metastases were associated with compromised survival for patients $<45$ years [18]. However, the results reported by Tran Cao et al. did not account for important potential confounders, including extent of tumor within the thyroid and extent of surgical resection. The study by Adam et al. reexamined this controversial issue in a large cohort from the NCDB. Lymph node metastases were associated with a $32 \%$ increase in risk of death in young patients. When the same analyses were applied to the SEER data, the investigators found a $29 \%$ increase in risk of death associated with metastases in younger patients. The effect of lymph node metastases among younger patients is comparable to the reported effect of lymph node metastases on survival among patients $\geq 45$ years [15]. This suggests that lymph node metastases potentially confer a similar prognostic impact in all adult patients with PTC, regardless of their age.

Adam et al. also demonstrated that the number of metastatic cervical lymph nodes was associated with incrementally compromised survival for up to six metastatic lymph nodes, after which more positive lymph nodes were not associated with additional risk of death. This finding signifies the prognostic importance of the number of lymph nodes harboring metastatic PTC in young patients and may argue for more rigorous preoperative screening. It also brings to light the question of whether there is a minimum number of lymph nodes that need to be removed to ensure reliable detection of lymph node metastases among patients undergoing cervical lymph node dissection.

While the findings from Adam et al. demonstrate a significant association between lymph node metastases and compromised survival among young patients, it is important to highlight that the reported absolute survival difference related to lymph node metastases was relatively small. This likely reflects the indolent nature of PTC and its excellent prognosis overall. This raises the question of whether a small absolute difference in survival should warrant consideration of more aggressive work-up and (potentially) management. However, the same thinking could be applied to lymph node metastases identified in patients $\geq 45$ years. In the literature, the absolute survival decrement conferred by the presence of lymph node metastases among patients $\geq 45$ years is relatively small. Zaydfudim et al. reported that lymph node metastases were associated with a comparable relative risk of death [15]. Additionally, the incidence of thyroid cancer is increasing faster than any other malignancy in the USA, such that it is expected to become the third most common cancer among women by 2019 [19]. Therefore, a small decrement in survival related to lymph 
node metastases would translate into a potentially larger number of death events at the population level.

The presence of lymph node metastases is an independent predictor for recurrence in PTC [20]. The JCO study unfortunately does not provide additional information with regard to the effect of lymph node metastases on risk of PTC recurrence due to unavailability of recurrence data in the NCDB or SEER. While disease recurrence is an important end point in differentiated thyroid cancer, the observed survival difference in young patients with lymph node metastases is a notable and impactful finding.

\section{Financial \& competing interests} disclosure

JA Sosa is a member of the Medullary Thyroid Cancer Registry Data Monitoring Committee funded by GlaxoSmithKline, NovoNordisk, Astra Zeneca and Eli Lilly; and a member of the AJCC Endocrine Expert Panel. The authors have no relevant affiliations or financial involvement with any organization or entity with a financial interest in or financial conflict with the subject matter or materials discussed in the manuscript. This includes employment, consultancies, honoraria, stock ownership or options, expert testimony, grants or patents received or pending, or royalties.

No writing assistance was utilized in the production of this manuscript.

\section{References}

1 Adam MA, Pura J, Goffredo P et al. Presence and number of lymph node metastases are associated with compromised survival for patients younger than age 45 years with papillary thyroid cancer. J. Clin. Oncol. 33(21), 2370-2375 (2015).

2 Byrd DR, Compton CC, Fritz AG, Green FL, Trotti A III. AJCC Cancer Staging Manual (Volume 649). Springer, NY, USA (2010).

3 Byar DP, Green SB, Dor P et al. A prognostic index for thyroid carcinoma. A study of the E.O.R.T.C. Thyroid Cancer Cooperative Group. Eur. J. Cancer. 15(8), 1033-1041 (1979).

4 Sherman SI, Brierley JD, Sperling M et al. Prospective multicenter study of thyroiscarcinoma treatment: initial analysis of staging and outcome. National thyroid cancer treatment cooperative study registry group.

The study findings are timely as the AJCC is reassessing staging guidelines for all malignancies, including differentiated thyroid cancer. The data published by Adam et al. confirm the prognostic significance of lymph node metastases among young patients with PTC and may challenge the widely accepted belief regarding the unique importance of a patient age cut-point of 45 years as a prognostic factor [18]. Important questions remain: is there an alternative age cut-point to 45 years where prognosis changes? Should we abandon the concept of age dichotomization as a prognostic factor in PTC altogether? More data are needed in this research space.

\section{Cancer 83(5), 1012-1021 (1998).}

5 Hay ID, Grant CS, Taylor WF, Mcconahey WM. Ipsilateral lobectomy versus bilateral lobar resection in papillary thyroid carcinoma: a retrospective analysis of surgical outcome using a novel prognostic scoring system. Surgery 102(6), 1088-1095 (1987).

6 Cady B, Rossi R. An expanded view of risk-group definition in differentiated thyroid carcinoma. Surgery 104(6), 947-953 (1988).

7 Dean DS, Hay ID. Prognostic indicators in differentiated thyroid carcinoma. Cancer Control 7(3), 229-239 (2000).

8 Hay ID, Bergstralh EJ, Goellner JR, Ebersold JR, Grant CS. Predicting outcome in papillary thyroid carcinoma: development of a reliable prognostic scoring system in a cohort of 1779 patients surgically treated at one institution during 1940 through 1989. Surgery 114(6), 1050-1057; discussion 1057-1058 (1993).

9 Edge SB, Byrd DR, Compton CC, Fritz AG, Greene FL, Trotti A. AJCC Cancer Staging Manual. Springer, NY, USA, 649 (2010).

10 Cady B, Sedgwick CE, Meissner WA, Bookwalter JR, Romagosa V, Werber J. Changing clinical, pathologic, therapeutic, and survival patterns in differentiated thyroid carcinoma. Ann. Surg. 184(5), 541-553 (1976).

11 Franssila KO. Prognosis in thyroid carcinoma. Cancer 36(3), 1138-1146 (1975).

12 Russell MA, Gilbert EF, Jaeschke WF. Prognostic features of thyroid cancer. A long-term followup of 68 cases. Cancer 36(2), 553-559 (1975).

13 Crile G, Jr., Hazard JB. Relationship of the age of the patient to the natural history and prognosis of carcinoma of the thyroid. Ann.
Surg. 138(1), 33-38 (1953).

14 Beahrs OH, Myers MH. AJCC Manual For Staging of Cancer. JB Lippincott Company, PA, USA (1983).

15 Hughes CJ, Shaha AR, Shah JP, Loree TR. Impact of lymph node metastasis in differentiated carcinoma of the thyroid: a matched-pair analysis. Head Neck 18(2), 127-132 (1996).

16 Zaydfudim V, Feurer ID, Griffin MR, Phay JE. The impact of lymph node involvement on survival in patients with papillary and follicular thyroid carcinoma. Surgery 144(6), 1070-1077; discussion 1077-1078 (2008).

17 Nixon IJ, Wang LY, Palmer FL et al. The impact of nodal status on outcome in older patients with papillary thyroid cancer. Surgery 156(1), 137-146 (2014).

18 Tran Cao HS, Johnston LE, Chang DC, Bouvet M. A critical analysis of the American Joint Committee on Cancer (AJCC) staging system for differentiated thyroid carcinoma in young patients on the basis of the Surveillance, Epidemiology, and End Results (SEER) registry. Surgery 152(2), 145-151 (2012).

19 Aschebrook-Kilfoy B, Kaplan EL, Chiu BC, Angelos P, Grogan RH. The acceleration in papillary thyroid cancer incidence rates is similar among racial and ethnic groups in the United States. Ann. Surg. Oncol. 20(8), 2746-2753 (2013).

20 Leboulleux S, Rubino C, Baudin E et al. Prognostic factors for persistent or recurrent disease of papillary thyroid carcinoma with neck lymph node metastases and/or tumor extension beyond the thyroid capsule at initial diagnosis. J. Clin. Endocrinol. Metab. 90 (10), 5723-5729 (2005). 